\title{
Behavior of sandwich panels in a fire
}

\author{
Eugenia Chelekova ${ }^{1, a^{*}}$ \\ ${ }^{1}$ National Research University "Moscow State University of Civil Engineering, Russia, 129337, \\ Moscow, Yaroslavskoye shosse, 26
}

\begin{abstract}
For the last decades there emerged a vast number of buildings and structures erected with the use of sandwich panels. The field of application for this construction material is manifold, especially in the construction of fire and explosion hazardous buildings. In advanced evacu-ation time calculation methods the coefficient of heat losses is defined with dire regard to fire load features, but without account to thermal and physical characteristics of building envelopes, or, to be exact, it is defined for brick and concrete walls with gross heat capacity. That is why the application of the heat loss coefficient expression obtained for buildings of sandwich panels is impossible because of different heat capacity of these panels from the heat capacities of brick and concrete building envelopes. The article conducts an analysis and calculation of the heal loss coefficient for buildings and structures of three layer sandwich panels as building envelopes.
\end{abstract}

\section{Introduction}

Three-layer sandwich panels are well-known in the construction of various facilities. Supermarkets, terminals, industrial buildings in the oil and gas industry are built using sandwich panels.

It is necessary to consider separately the behavior of sandwich panels in a fire. This is due to their distinctive features. If the fire appears small thermal inertia of the panels.

\section{Materials and Methods}

The time allotted for the evacuation of people in case of fire, largely depends on heat loss in the building envelope. Currently, these losses are determined by the ratio of heat losses [1]. The method of determining this factor is designed to brick and concrete walling [1-2] and without reason, applied to sandwich panels. In this article we attempt to determine the coefficient of heat loss from the hot gases in the sandwich panel. The study is limited to the initial stages of fire development, which lasts no more than 20 minutes. When the fire heats up only the metal sheet in contact only with hot gases. The flow of heat in the insulation at

\footnotetext{
*Corresponding author: Kiara_lion@mail.ru
} 
the considered stage of the fire is missing. Sheet metal thickness $\delta=0.5-1 \mathrm{~mm}$ is thermally thin body and its temperature is uniform in thickness. The temperature change of the sheet in this case is described by the equation:

$$
t_{1} \frac{d T}{d t}=\left(T_{s}(t)-T(t)\right.
$$

$t_{1}=\frac{\delta \rho_{m} C_{p m}}{\alpha}-$ the characteristic time of temperature change of the sheet, $\delta$ - is the thickness of the sheet, $C_{p m}$ and $\rho_{m}$ - heat capacity the density of the sheet material, $\alpha$-is coefficient of heat transfer from the gaseous medium to the structure, $T_{s}(t)$ - is the volumetric average temperature of the gas environment in the fire, $T(t)$ - is the temperature of the sheet is determined by:

$$
T(t)=T_{0} e^{-\frac{t}{t_{1}}}+\frac{1}{t_{1}} \int_{0}^{t} T_{s}(\tau) e^{-\frac{t-\tau}{t_{1}}} d \tau
$$

$T_{0}$ - the initial temperature of the medium and the sheet. Consider the temperature change of the environment according to the law:

$$
T_{c}(t)=T_{0}\left(1+\frac{t}{t_{s}}\right)
$$

which corresponds to the first approximation for the case of a fire with a constant burning area. [3].

Where $t_{s}=\frac{V_{0} \rho_{0} T_{0} C_{p}}{Q_{n} \psi F_{g}(1-\varphi)}$ the characteristic time of measurement of the temperature

of the gaseous medium in this case. $\mathrm{V}_{0}$ - is the volume of the room, $\rho_{0}-$ is the initial density of the gaseous medium, $C_{p 0}$ - heat capacity of air, $Q_{n}$ - is the heat of combustion of combustible material, $\psi$ - specific rate of burnout, $F_{g}$ - the area of the fire, $\varphi(\mathrm{t})-$ coefficient of heat loss to the time t:

$$
\varphi=\frac{q_{\sum(t)}}{Q_{P(t)}}
$$

$\mathrm{q} \Sigma_{(\mathrm{t})}$ - is the amount of heat transferred from the environment in the building envelope area $\mathrm{F}_{\mathrm{P}}$ - time t. $\mathrm{Q}_{\mathrm{p}}$ - is the energy released by fire for time $\mathrm{t}$.

$$
\begin{aligned}
& T(t)=\left(\frac{t_{1}}{t_{s}} e^{-{ }^{-\frac{t}{t_{1}}}}+1-\frac{t_{1}}{t_{s}}+\frac{t}{t_{s}}\right) T_{0} \\
& q_{\Sigma(t)}=C p_{m} M\left(T(t)-T_{0}\right) ;
\end{aligned}
$$

$M=F_{P} \rho_{m} \delta$ - is the total mass of the metallic inner coating. As a result, the ratio of heat loss is equal to:

$$
\varphi=\frac{C p_{m} M\left(1+\frac{t_{1}}{t} e^{-\frac{t}{t_{1}}}-\frac{t_{1}}{t}\right)}{V_{0} \rho_{0}+C_{p m} M\left(1+\frac{t_{1}}{t} e^{-\frac{t}{t_{1}}}-\frac{t_{1}}{t}\right)}
$$


Figure 1 presents the dependence of coefficient of heat losses for different values of the parameter: $P=\frac{C_{p m} M}{V_{0} \rho_{0} C_{p}}$.

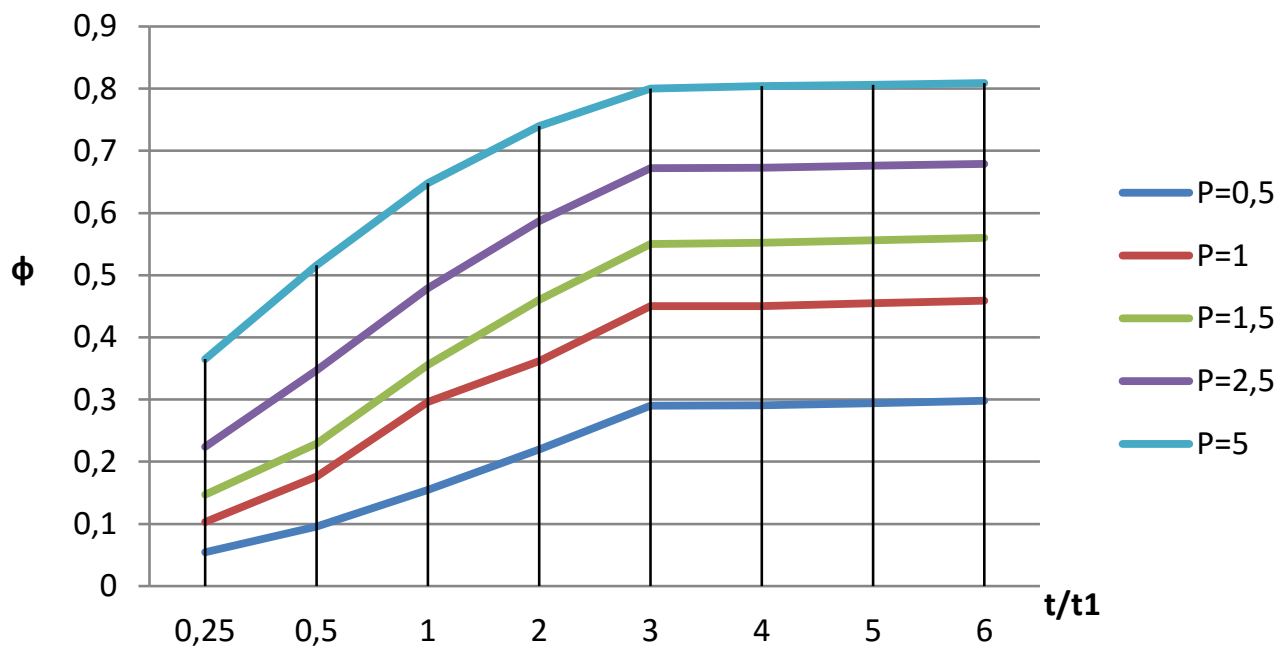

Fig. 1. The dependence of coefficient of heat losses for different values of the parameter «P».

The parameter $« \mathrm{P} »$ is proportional $\delta / V_{0}^{1 / 3}$.

For sheet metal: $P=\frac{3093 G \delta}{V_{0}^{1 / 3}}$

Here $« \mathrm{G} »$ is defined as $\mathrm{F}_{\mathrm{p}}=\mathrm{GV}_{0}{ }^{2 / 3}$ and depends on the shape of the room.

From Fig.1 shows that with the decrease of the parameter $\langle\mathrm{P} »$ decreases and the coefficient of heat loss. Thus determined the dependence $\varphi$ sheet thickness and volume of the room. It should be noted that in the approximation of a thermally thin walling coefficient of heat loss does not depend on properties of the combustible material and the heat transfer coefficient $\alpha$ is only in the scale of time t.

Brick and concrete wall thickness $\delta>5 * 10^{-2} \mathrm{~m}$ for the initial stage of the fire $<10^{3} \mathrm{sec}$, is thermally thick solids. This means that the conditions on the opposite side do not affect the heat transfer process. For thermally thick solids, the surface temperature $T_{1}$ in the case of constant temperature $T_{S}$ and with a constant heat transfer coefficient $\alpha$ varies with time according to [4].

$$
T_{1}(t)-T_{s}=\left(T_{s}-T_{0}\right)\left[1-\exp \left(\frac{t}{t_{2}}\right) \operatorname{erfc}\left(\frac{t}{t_{2}}\right)^{1 / 2}\right]
$$

$t_{2}=\frac{C_{p} \rho \lambda}{\alpha^{2}}$ is the characteristic time of change of surface temperature. Brick walls with $\alpha=12 \mathrm{Vt} / \mathrm{m}^{2} \mathrm{~K}, \mathrm{t}_{2}=8680 \mathrm{sec}$. For times $t / t_{2}<0,15 \mathrm{sec}$. the expression (7) can be replaced by the expression (5): 


$$
\left(T_{1}-T_{0}\right)=\left(T_{s}-T_{0}\right) \frac{\left(\gamma \frac{t}{t_{2}}\right)^{1 / 2}}{1+\left(\gamma \frac{t}{t_{2}}\right)^{1 / 2}}
$$

Where $\gamma=\frac{4}{\pi}$.

For variable temperature environment: $T_{s}=T_{0}\left(1+\frac{t}{t_{s}}\right)$ heat flux at the wall is determined.

These structures are thermally thick plates as long as the fire do not reach their critical values. For such structures the heat flow at constant temperature, the gas environment of the $T_{\mathrm{s}}$ and constant heat transfer coefficient $\alpha$ from the environment to the structure is equal to [3]

$$
q_{\Sigma 2}=\frac{\alpha t^{2} F_{p} T_{0}}{2 t_{s}}\left[1-\frac{8}{15}\left(\gamma \frac{t}{t_{2}}\right)^{1 / 2}\right]
$$

In this case $t_{s}=\frac{V_{0} \rho_{0} T_{0} C_{p}}{Q_{n} \psi F_{g}\left(1-\varphi_{2}\right)}$, where $\varphi_{2}-$ coefficient of heat loss in a brick wall for time t.

Coefficient of heat loss is equal to:

$$
\varphi_{2}=\frac{\alpha F_{P} t\left[1-\frac{8}{15}\left(\gamma \frac{t}{t_{2}}\right)^{1 / 2}\right]}{2 V_{0} \rho_{0} C_{p}+\alpha F_{P} t\left[1-\frac{8}{15}\left(\gamma \frac{t}{t_{2}}\right)\right]}
$$

\section{Results}

Table 1 shows for comparison the dependence $\varphi_{2}(t)$ of $\left(t / t_{1}\right)$ for different time scales $t_{1}$, that is, the brick wall is compared with sheet metal of different thickness with the same amount of space. From the comparison it is seen that the heat loss in a sandwich panel is always less than a brick wall. The maximum difference corresponds to the double and large rooms $10^{4}$ $\mathrm{m}^{3}$ and small thicknesses $\approx 0.5 \mathrm{~mm}$.

Figure 2 gives the opportunity to cross-section coordinate is $t / t_{1}=$ const. to determine the dependence $\varphi_{1}(t)$ of the volume $V_{0}$ for different ages.

Table 1. The dependence $\varphi_{2}(t)$ of $\left(t / t_{1}\right)$ for different time scales $t_{1}$.

\begin{tabular}{|c|c|c|c|c|c|c|c|c|c|c|}
\hline \multicolumn{2}{|c|}{$t / t_{1}$} & $\mathbf{0 , 2 5}$ & $\mathbf{0 , 5}$ & $\mathbf{1}$ & $\mathbf{2}$ & $\mathbf{3}$ & $\mathbf{4}$ & $\mathbf{5}$ & $\mathbf{6}$ & $\infty$ \\
\hline \multirow{2}{*}{$\mathbf{P} / \mathbf{0 , 5}$} & panel & 0,0544 & 0,096 & 0,155 & 0,220 & 0,290 & 0,291 & 0,294 & 0,298 & 0,333 \\
\cline { 2 - 12 } & brick & 0,0570 & 0,106 & 0,019 & 0,310 & 0,400 & 0,465 & 0,515 & 0,560 & - \\
\hline \multirow{2}{*}{$\mathbf{1}$} & panel & 0,103 & 0,176 & 0,296 & 0,362 & 0,450 & 0,450 & 0,455 & 0,459 & 0,500 \\
\cline { 2 - 11 } & brick & 0,108 & 0,192 & 0,320 & 0,475 & 0,570 & 0,634 & 0,660 & 0,715 & - \\
\hline
\end{tabular}




\begin{tabular}{|c|c|c|c|c|c|c|c|c|c|c|}
\hline $\mathbf{1 , 5}$ & panel & 0,147 & 0,229 & 0,356 & 0,460 & 0,550 & 0,552 & 0,556 & 0,560 & 0,600 \\
\cline { 2 - 11 } & brick & 0,154 & 0,264 & 0,412 & 0,576 & 0,670 & 0,723 & 0,760 & 0,790 & 1,00 \\
\hline \multirow{2}{*}{, 5} & panel & 0,224 & 0,347 & 0,479 & 0,587 & 0,672 & 0,673 & 0,676 & 0,679 & 0,714 \\
\cline { 2 - 11 } & brick & 0,232 & 0,373 & 0,540 & 0,693 & 0,770 & 0,810 & 0,840 & 0,860 & 1,00 \\
\hline \multirow{2}{*}{$\mathbf{5}$} & panel & 0,365 & 0,516 & 0,648 & 0,740 & 0,800 & 0,804 & 0,806 & 0,809 & 0,833 \\
\cline { 2 - 10 } & brick & 0,377 & 0,540 & 0,700 & 0,820 & 0,870 & 0,900 & 0,914 & 0,926 & 1,00 \\
\hline
\end{tabular}

\section{Conclusion}

The characteristic time value $t_{1}$ for the initial stage of the fire $\alpha \approx 12-16 \mathrm{Vt} / \mathrm{m}^{2} \mathrm{~K}$ is $100-300$ seconds, depending on the thickness of the sheet. By the end of the initial stage of fire when the indoor temperature reaches a critical value for the conditions of the evacuation ratio $\left(t / t_{1}\right)$ is 2-6 times. In Fig. 2 shows the dependence $\varphi_{1}(\mathrm{t})$ from the volume of the room for occasions: 1) $t / t_{1}=2$ and 2$) t / t_{1}=6$.

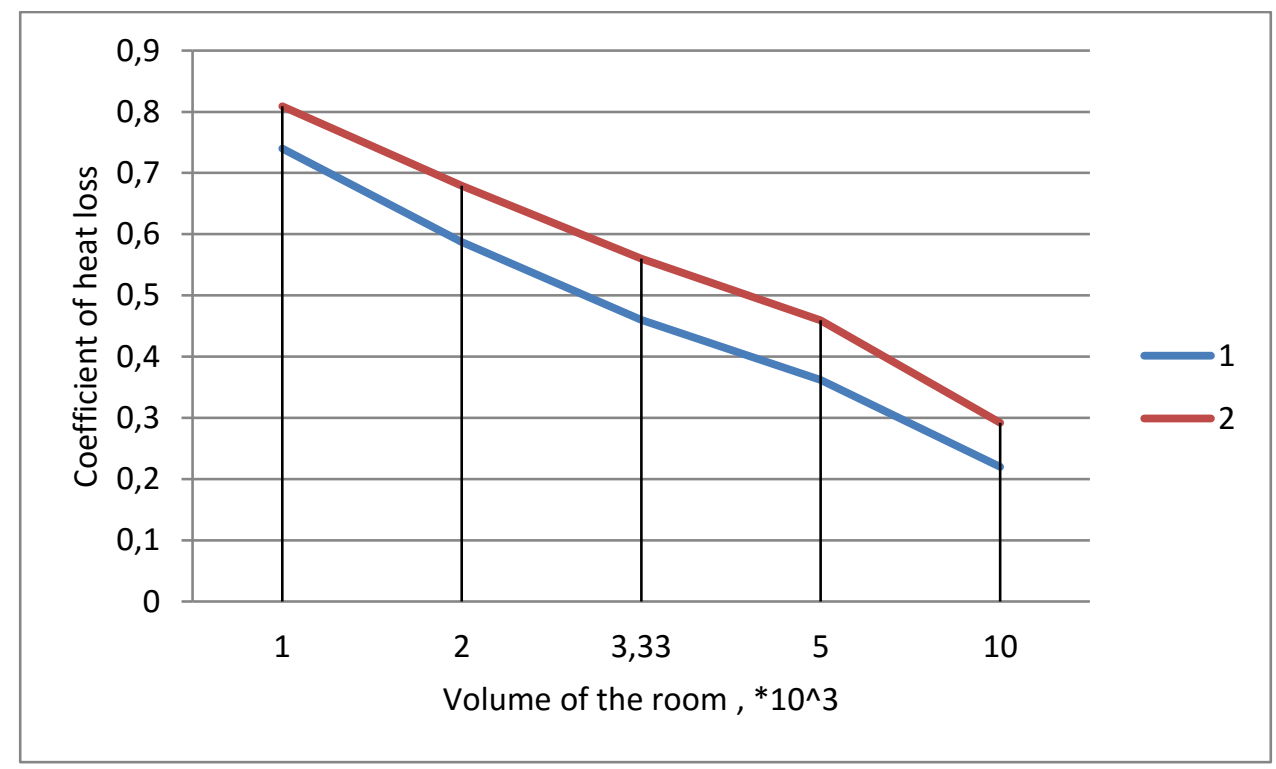

Fig. 2. The dependence $\varphi_{1}(t)$ from the volume of the room for occasions: 1$) t / t_{1}=2$ and 2) $t / t_{1}=6$.

The expression (9) is derived from (8) for changing the temperature of the environment using the theory of Duamel's [6]. The presented method can be used for the case of arbitrary changes in ambient temperature. The use of Duamel's theorem is due to numerical integration.

\section{References}

1. Yu. Koshmarov, Forecasting of fire hazards in the case of indoor fire. Moscow, State Fire Academy of Ministry of Interior of Russia Publ, (2000)

2. Methodology og the calculated values of fire risk in buildings, facilities and structures of various classes of functional fire hazard: Annex to the Order of the Russian Emergencies Ministry 30.06.2009 №382. Russian Newspaper, (2009)

3. Ye. Salymova, Dynamics of development of dangerous factors in buildings with enclosing structures made of sandwich panels in fires and explosions Candidate 
dissertation. 05.26.03/ Salymova Ye. Yu. Moscow State University of Civil Engineering. Moscow ( 2015 )

4. A. Lykov, Theory of thermal conductivity. Moscow, Vysshaya shkola Publ., (1967)

5. V.A.Gore, M. Fomina, Simplified calculation of heat on a flat surface. Pozharovzryvobezopasnost - Fire and Explosion Safety, 2016,vol.25, no.3, pp 5-14.

6. A.N. Tihonov, A.A. Samarskiy Equations of mathematical physics. Moscow, Nauka Publ. 1977 - 735 p. 